OPEN ACCESS

Edited by:

Roberto Esposito,

A.O. Ospedali Riuniti Marche

Nord, Italy

Reviewed by:

Feng Liu,

Tianjin Medical University General

Hospital, China

Jiuquan Zhang,

Chongqing University,

China

*Correspondence:

Fuhua Yan

yfh11655@rih.com

Shikun Zhan

zsk10715@rih.com

${ }^{t}$ These authors have contributed equally to this work

Specialty section: This article was submitted to Neuroimaging and Stimulation,

a section of the journal

Frontiers in Psychiatry

Received: 18 October 2019 Accepted: 24 December 2019

Published: 31 January 2020

Citation:

Li J, Gong $H, X u H$, Ding $Q$, He N, Huang $Y$, Jin $Y$, Zhang $C$, Voon $V$, Sun B, Yan F and Zhan S

(2020) Abnormal Voxel-Wise Degree Centrality in Patients

With Late-Life Depression: A

Resting-State Functional Magnetic

Resonance Imaging Study.

Front. Psychiatry 10:1024.

doi: 10.3389/fpsyt.2019.01024

\section{Abnormal Voxel-Wise Degree Centrality in Patients With Late-Life Depression: A Resting-State Functional Magnetic Resonance Imaging Study}

\author{
Jun $\mathrm{Li}^{1 \dagger}$, Hengfen Gong ${ }^{2 \dagger}$, Hongmin $\mathrm{Xu}^{3 \dagger}$, Qiong Ding ${ }^{4}$, Naying $\mathrm{He}^{3}$, Ying Huang ${ }^{2}$, \\ Ying $\mathrm{Jin}^{2}$, Chencheng Zhang ${ }^{1}$, Valerie Voon ${ }^{5}$, Bomin Sun ${ }^{1}$, Fuhua Yan ${ }^{3 *}$ \\ and Shikun Zhan ${ }^{1 *}$ \\ 1 Department of Functional Neurosurgery, Ruijin Hospital, Shanghai Jiao Tong University School of Medicine, Shanghai, \\ China, ${ }^{2}$ Department of Psychiatry, Shanghai Pudong New Area Mental Health Center, Tongji University School of Medicine, \\ Shanghai, China, ${ }^{3}$ Department of Radiology, Ruijin Hospital, Shanghai Jiao Tong University School of Medicine, Shanghai, \\ China, ${ }^{4}$ Neural and Intelligence Engineering Center, Institute of Science and Technology for Brain-Inspired Intelligence, Fudan \\ University, Shanghai, China, ${ }^{5}$ Department of Psychiatry, University of Cambridge, Cambridge, United Kingdom
}

Objectives: Late-life depression (LLD) has negative impacts on somatic, emotional and cognitive domains of the lives of patients. Elucidating the abnormality in the brain networks of LLD patients could help to strengthen the understanding of LLD pathophysiology, however, the studies exploring the spontaneous brain activity in LLD during the resting state remain limited. This study aimed at identifying the voxel-level whole-brain functional connectivity changes in LLD patients.

Methods: Fifty patients with late-life depression (LLD) and 33 healthy controls were recruited. All participants underwent a resting-state functional magnetic resonance imaging scan to assess the voxel-wise degree centrality (DC) changes in the patients. Furthermore, DC was compared between two patient subgroups, the late-onset depression (LOD) and the early-onset depression (EOD).

Results: Compared with the healthy controls, LLD patients showed increased DC in the inferior parietal lobule, parahippocampal gyrus, brainstem and cerebellum $(p<0.05$, AlphaSim-corrected). LLD patients also showed decreased DC in the somatosensory and motor cortices and cerebellum ( $p<0.05$, AlphaSim-corrected). Compared with EOD patients, LOD patients showed increased centrality in the superior and middle temporal gyrus and decreased centrality in the occipital region $(p<0.05$, AlphaSim-corrected). No significant correlation was found between the DC value and the symptom severity or disease duration in the patients after the correction for multiple comparisons.

Conclusions: These findings indicate that the intrinsic abnormality of network centrality exists in a wide range of brain areas in LLD patients. LOD patients differ with EOD patients 


\section{in cortical network centrality. Our study might help to strengthen the understanding of the pathophysiology of LLD and the potential neural substrates underlie related emotional and cognitive impairments observed in the patients.}

Keywords: late-life depression, resting state, functional magnetic resonance imaging, degree centrality, onset age

\section{INTRODUCTION}

Depression refers to a mental disorder characterized by low mood present across most situations for at least two weeks. Latelife depression (LLD) can be defined as a major depressive episode occurring in old age, usually over 60 years of age. Aside from the emotional and somatic burdens associated with depression, such as insomnia, anorexia and fatigue (1), elderly depressive patients may also show impairment in various cognitive domains including attention $(2)$, memory $(3,4)$, information processing speed $(5,6)$, and executive functions $(4,7)$. All these somatic, emotional, and cognitive abnormalities may severely affect the life quality of the patients.

Elucidating the brain abnormality of LLD patients could help to strengthen the understanding of LLD pathophysiology, and develop effective interventions. Grey matter alterations have been reported in multiple brain areas including the frontal, parietal regions and limbic system in LLD patients $(8,9)$. Several taskbased functional magnetic resonance imaging (fMRI) studies have also indicated abnormal functional activities in the areas of frontal lobes and limbic system $(10,11)$. While structural alterations reflect the long-term influence of depression, and task-based imaging profiles the altered brain reaction to external stimuli or under specific situations, resting-state fMRI explores the intrinsic changes of brain activity in the state without any influence of external stimuli. Resting-state fMRI studies using the method of independent component analysis has revealed that the default mode network, the frontoparietal network and the sensorimotor network showed abnormal connectivity in LLD patients $(12,13)$. LLD patients also exhibited abnormal local synchronization in various brain areas, as revealed by the measure of regional homogeneity (14). These observations suggest that the brain activities in the resting state have extensive cortical and subcortical abnormalities in LLD patients, which might be associated with clinical manifestations such as emotional disturbance and cognitive deficits observed in the patients.

Degree centrality (DC), an index of the total weights of connections for a given node, has recently been applied to reveal the core hub architecture of brain networks (15). Increased voxel-wise DC in a brain region indicates an elevated degree of its global connectivity, and decreased voxelwise DC in a brain region suggests a reduced degree of its global connectivity. Voxel-wise DC has been applied to reveal the

\footnotetext{
Abbreviations: DC, degree centrality; EOD, early-onset depression; fMRI, functional magnetic resonance imaging; HAMD, Hamilton Depression Scale; HC, healthy control; LLD, late-life depression; LOD, late-onset depression; MMSE, Mini-Mental State Examination.
}

abnormal brain networks in various types of neurological or psychiatric diseases (16-22). This method has also been used to compare the brain network features of different psychiatric diseases with potentially similar neural pathology, such as autism and attention-deficit hyperactivity disorder (23), or to examine the brain-network difference between the subtypes of a disease such as the Parkinson's disease patients with depression and those without depression (24). The alterations of the wholebrain degree centrality in young patients with major depression have been illustrated in two recent studies revealing that the frontoparietal network, the limbic system, and the striatal areas show DC abnormality in the patients (25). However, depressionspecific alterations of network centrality among LLD patients yet remain to be identified.

LLD can be divided into the early-onset depression (EOD, depression occurred for the first time under the age of 60) and late-onset depression (LOD, depression occurred for the first time over the age of 60) according to the onset age of depression (most studies use 60 years as the onset age to distinguish EOD and LOD). While some studies indicate that the LOD patients are not different from the EOD patients in most aspects such as etiological factors, phenomenology or clinical outcomes (26), other studies suggest that the LOD patients show a more severe emotional burden and more extensive cognitive impairments than EOD patients (27-29). Several imaging studies have indicated differences in structure or task-based functional activity between EOD and LOD patients in a wide range of brain areas, including the medial and lateral frontal areas, hippocampus and amygdala (3035). However, only a few studies have examined the difference in resting-state functional network between EOD and LOD patients. One study using regional homogeneity and the other study using amplitude of low-frequency fluctuation as the indices suggest the local synchronization and low-frequency fluctuation differ in multiple cortical areas and brainstem between LOD and EOD patients $(36,37)$. The centrality profile of the resting-state brain networks has not been elucidated between LOD and EOD patients.

In the present study, we hypothesized that the architecture of brain networks reflected by degree centrality may show abnormality in cortical and subcortical areas in the LLD patients.

To explore the alterations of functional centrality in LLD patients, we examined the difference in voxel-wise DC between the LLD patients and healthy controls (HCs). To further examine the hypothesis that a difference of the architecture of brain networks may exist between EOD and LOD patients, we compared the DC between the two subgroups of patients. The relationship between the centrality indices of the brain areas being identified abnormal and the clinical assessment was also examined. 


\section{METHODS}

All procedures used in the present study were approved by the Ethics Committee of Shanghai Pudong New Area Mental Health Center (approval letter: PDJWLL2014001). Patients with LLD and HCs were recruited via advertisements and were fully instructed regarding experimental procedures. All participants gave their written informed consent in accordance with the Declaration of Helsinki.

\section{Participants}

Patients diagnosed with major depression according to the criteria in the International Classification of Diseases (ICD-10) by a physician were recruited at Pudong New Area Mental Health Center in Shanghai, China. Healthy volunteers whose age matched to \pm 10 years with the patients were recruited as controls. Participants aged under 60 or over 80 , with a history of severe head trauma, with alcohol abuse, with psychiatric diseases other than depression, with claustrophobia, or with metal or electronic implants were excluded. Patients were using tricyclic, selective serotonin reuptake inhibitors or serotonin and noradrenaline reuptake inhibitors when they were recruited.

\section{Demographics and Clinical Assessment}

Basic demographics (i.e. gender, age, education level, handedness) of the participants were collected. Both the patients and HCs filled out the Hamilton Depression Scale (HAMD) (38) right before the resting-state fMRI scan. Patients were asked to report the onset age and the duration of depression. In addition, Mini-Mental State Examination (MMSE) [Folstein, (39)] was carried out and potential participants scored lower than 21, who may have moderate to severe cognitive impairment, were excluded from the study. Mann-Whitney test was used for the between-group comparisons of age, disease duration, and scores of HAMD and MMSE. Chi-square test was used for the between-group comparisons of gender and education level.

\section{Image Acquisition}

Resting-state fMRI was performed on a GE Signa HDxt 3.0 T MRI scanner using an eight-channel phased-array head coil. Each participant lay supine with their head snugly fixed by foam pads. The participant was asked to keep still as long as possible and to keep his/her eyes closed but remain awake. Resting-state fMRI was obtained using an echo-planar imaging sequence with protocols of TR $=2000 \mathrm{~ms}, \mathrm{TE}=30 \mathrm{~ms}$, flip angle $=90^{\circ}$, FOV 240 $\mathrm{mm} \times 240 \mathrm{~mm}$, matrix $=64 \times 64$, voxel size $3.75 \mathrm{~mm} \times 3.75$ $\mathrm{mm} \times 4.00 \mathrm{~mm}, 35,37$ or 39 axial slices, 210 volumes acquired in $7 \mathrm{~min}$.

\section{Imaging Data Preprocessing}

Preprocessing of resting-state fMRI data was conducted using Data Processing Assistant for Resting-State fMRI (DPARSF; http://rfmri.org/dparsf) software (version 4.5) embedded in Data Processing and Analysis for Brain Imaging (DPABI; http://rfmri.org/dpabi) toolbox (version 4.1) on the MATLAB platform (MathWorks, Natick, MA, USA). In brief, the first 10 volumes of each functional time series were discarded, as the participants were adjusting themselves to the fMRI environment during that period. The remaining 200 images were slice-timecorrected with the 35th, 37th or 39th slice as the reference and spatially realigned for head motion. Head motion was assessed by evaluating three translations and three rotations for each scan. Translational thresholds were set to $\pm 3 \mathrm{~mm}$, while rotational thresholds were limited to $\pm 3^{\circ}$. After head motion correction, functional images were spatially normalized to Montreal Neurological Institute (MNI) space using echo-planar imaging sequence templates (resampled voxel size $3 \mathrm{~mm} \times 3 \mathrm{~mm} \times 3 \mathrm{~mm}$ ). All images were linearly detrended and bandpass-filtered (0.01$0.1 \mathrm{~Hz}$ ) to have the high-frequency respiratory and cardiac noises reduced. The white matter signal, cerebrospinal fluid signal, and Friston 24 head motion parameters were regressed out from the time courses of every voxel.

\section{Voxel-Wise Degree Centrality}

The value of degree centrality was calculated using DPARSF (http://rfmri.org/dparsf). For each voxel, the blood-oxygen-leveldependent (BOLD) time course was extracted and the Pearson correlation coefficients with every other voxel in the brain were calculated. A matrix of Pearson correlation coefficients between any pair of voxels was obtained to construct the whole-brain functional connectivity matrix for each participant. Finally, the resulting matrices (DC maps) were smoothed with a Gaussian kernel (full-width half maximum $=6 \mathrm{~mm}$ ) to enable group comparisons.

To obtain the spatial distribution of DC maps for the HC group and the LLD group, the averaged DC map was calculated for each group using the image calculator module embedded in DPABI. The resulting averaged DC maps were overlaid on rendering views with BrainNet Viewer (http://www.nitrc.org/ projects/bnv).

To determine the abnormality in core brain hub architecture as reflected by DC in the LLD patient group, we identified the clusters with DC difference between the LLD patients and HCs, using two-sample $t$-test with gender, age, education level, score of MMSE and framewise displacement regressed out. To determine the difference of DC between LOD patients and EOD patients, another two-sample $t$-test was performed between the LOD group and EOD group in the same manner. AlphaSim correction was used for multiple comparisons to achieve a corrected $p<0.05$ determined by the Monte Carlo simulation by a combination of a voxel-wise threshold of $p<0.001$ and a minimum cluster size calculated by the AlphaSim program embedded in DPABI (https://afni.nimh.nih.gov/pub/dist/doc/ manual/AlphaSim.pdf), The resulting $t$ maps were overlaid on rendering views with BrainNet Viewer and on axial views in slices with the viewer module embedded in DPABI (http://rfmri. org/dpabi), and the anatomy of surviving brain regions was reported using xjView software (http://www.alivelearn. net/xjview).

To further examine whether there exists a relationship between the abnormal DC values identified in the patients and the related clinical assessment (onset age of depression, duration of depression and HAMD score), Pearson's correlation was 
respectively calculated in the LLD group and two subgroups. The averaged DC values of abnormal brain regions for the relationship analyses were extracted using spheres of 6-mm radius centering at the coordinates with peak statistical difference from the between-group comparisons (LLD group vs. HC group, or LOD group vs. EOD group) using the signal extractor module embedded in DPABI. To control for multiple comparisons, we used a Bonferroni correction of $p=0.016$ as the significant statistical threshold (three correlations were examined for each spherical region of interest).

\section{RESULTS}

\section{Demographics and Clinical Assessment of Participants}

The detailed demographics and results of the clinical assessment of the participants are illustrated in Table 1. The 50 LLD patients (19 males, 31 females) had a mean age of $66.6 \pm 0.7$ (range: $60-78$ ) years, $84.0 \pm 17.2$ months of disease duration, mean scores of $21.9 \pm 1.5$ on HAMD and $28.1 \pm 0.3$ on MMSE. The 33 HCs (17 males, 16 females) had a mean age of $67.2 \pm 0.8$ (range: 60-78) years. All participants were right-handed. The gender, age and mean score on MMSE were not significant between the patients and healthy controls $(p s>0.091)$. The education level $(p=0.043)$ and score on HAMD $(p<0.001)$ differed significantly between the groups.

The demographics and clinical assessment of EOD patients and LOD patients are also illustrated in Table 1. The two subgroups of patients did not differ in gender, education, scores on HAMD or MMSE ( $p s>0.079)$. There was a significant between-group difference of age (LOD: $68.5 \pm 1.0$, EOD: $64.0 \pm 0.7, p=0.002)$. Gender, age, education level and score on MMSE were used as covariates in the between-group

TABLE 1 | Demographics and clinical assessment of the participants.

\begin{tabular}{|c|c|c|c|c|}
\hline & \multicolumn{2}{|c|}{ LLD Patients } & $\mathrm{HC}$ & $p$ value \\
\hline Numbers & \multicolumn{2}{|c|}{50} & 33 & \\
\hline Gender (M/F) & \multicolumn{2}{|c|}{$19 / 31$} & $17 / 16$ & 0.224 \\
\hline Age (year) & \multicolumn{2}{|c|}{$66.6 \pm 0.7$} & $67.2 \pm 0.8$ & 0.499 \\
\hline $\begin{array}{l}\text { Education } \\
\text { (I/E/S/H/C) }\end{array}$ & \multicolumn{2}{|c|}{$1 / 5 / 32 / 6 / 6$} & $1 / 4 / 10 / 8 / 10$ & $0.043^{\star}$ \\
\hline Onset age (year) & \multicolumn{2}{|c|}{$59.6 \pm 1.6$} & NA & \\
\hline Duration (month) & \multicolumn{2}{|c|}{$84.0 \pm 17.2$} & NA & \\
\hline HAMD-24 & \multicolumn{2}{|c|}{$21.9 \pm 1.5$} & $2.4 \pm 0.6$ & $<0.001^{*}$ \\
\hline \multirow[t]{2}{*}{ MMSE } & \multicolumn{2}{|c|}{$28.1 \pm 0.3$} & $28.9 \pm 0.2$ & 0.091 \\
\hline & LOD patients & EOD patients & $p$ value & \\
\hline Numbers & 29 & 21 & & \\
\hline Gender (M/F) & $14 / 15$ & $5 / 16$ & 0.079 & \\
\hline Age (year) & $68.5 \pm 1.0$ & $64.0 \pm 0.7$ & $0.002^{*}$ & \\
\hline Education & $1 / 2 / 16 / 5 / 5$ & 0/3/16/1/1 & 0.249 & \\
\hline \multicolumn{5}{|l|}{ (I/E/S/H/C) } \\
\hline Onset age (year) & $66.7 \pm 1.0$ & $49.9 \pm 2.3$ & $<0.001^{*}$ & \\
\hline Duration (month) & $22.4 \pm 4.8$ & $169.1 \pm 32.5$ & $<0.001^{\star}$ & \\
\hline HAMD-24 & $21.7 \pm 2.1$ & $22.3 \pm 2.3$ & 0.911 & \\
\hline MMSE & $28.2 \pm 0.4$ & $28.0 \pm 0.5$ & 0.980 & \\
\hline
\end{tabular}

$C$, college and above; $E$, elementary school; EOD, early-onset depression; $F$, female; HAMD, H, high school; Hamilton Depression Scale; HC, healthy control; I, illiteracy; LOD, late-onset depression; M, male; MMSE, Mini-Mental State Examination; p, probability; S, secondary school. * indicates significant difference $(p<0.05)$ between the groups. comparisons of DC maps, in order to exclude the potential impact from demographics.

\section{Difference Between LLD Patients and $\mathrm{HCs}$}

The mean framewise displacement of the LLD group $(0.20 \pm$ $0.02 \mathrm{~mm})$ was slightly larger than that of the HC group $(0.14 \pm$ $0.01 \mathrm{~mm}, p=0.009$ ), and thus was also used as a covariate in the statistical analyses of DC. DC maps for the HC group and the LLD group are presented in Figure 1. The estimated smoothness of the DC map is FWHMx $=6.203 \mathrm{~mm}, \mathrm{FWHMy}=6.402 \mathrm{~mm}$, FWHMz $=6.169 \mathrm{~mm}, \mathrm{dLh}=0.512$. AlphaSim correction is performed with a combination of voxel $p<0.001$ and cluster size $>12$. Compared with the HCs, the LLD patients showed increased DC in the right inferior parietal lobule, right parahippocampal gyrus, right cerebellum and bilateral brainstem ( $p<0.05$, AlphaSim-corrected, Figure 2, Table 2). The LLD patients showed decreased DC in the brain areas of the left precentral and postcentral gyri, and the left cerebellum ( $p<0.05$, AlphaSim-corrected, Figure 2, Table 2).

The result with AlphaSim correction by a combination of voxel $p<0.01$ and cluster size $>29$ is also presented in the supplementary material (Figure S1, Table S1) for reference only, in case some brain areas with potential differences couldn't survive the voxel p level of 0.001 due to the relatively small sample size.

\section{Difference Between LOD Patients and EOD Patients}

Brain areas with a statistical difference in DC between the LOD patients and EOD patients were examined using between-group comparison. The mean framewise displacement of the LOD patient group $(0.14 \pm 0.02 \mathrm{~mm})$ was not different from that of the EOD patient group $(0.15 \pm 0.02 \mathrm{~mm}, p=0.158)$. The estimated smoothness of the DC map is FWHMx $=6.123 \mathrm{~mm}$, FWHMy $=6.306 \mathrm{~mm}, \mathrm{FWHMz}=6.065 \mathrm{~mm}, \mathrm{dLh}=0.539$. AlphaSim correction is performed with a combination of voxel $p<0.001$ and cluster size $>12$. Compared with the EOD patient group, the LOD patient group showed increased DC in the area of right superior and middle temporal gyri $(p<0.05$, AlphaSimcorrected, Figure 3, Table 3). Decreased DC was found in the right cuneus in the LOD patients, compared with the EOD patients ( $p<0.05$, AlphaSim-corrected, Figure 3, Table 3).

The result with AlphaSim correction by a combination of voxel $p<0.01$ and cluster size $>28$ is also presented in the supplementary material (Figure S2, Table S2) for reference only.

\section{Relationship of DC With Clinical Assessment}

The relationship of DC with clinical assessment was explored by the calculation of Pearson's correlation. No significant correlation was found between the DC and the clinical assessment values (onset age of depression, disease duration or HAMD score) after the Bonferroni correction for multiple comparisons in the LLD patient group ( $p s>0.094$ ), or in the two subgroups (LOD: $p s>0.056$; EOD group: $p s>0.094$ ). 


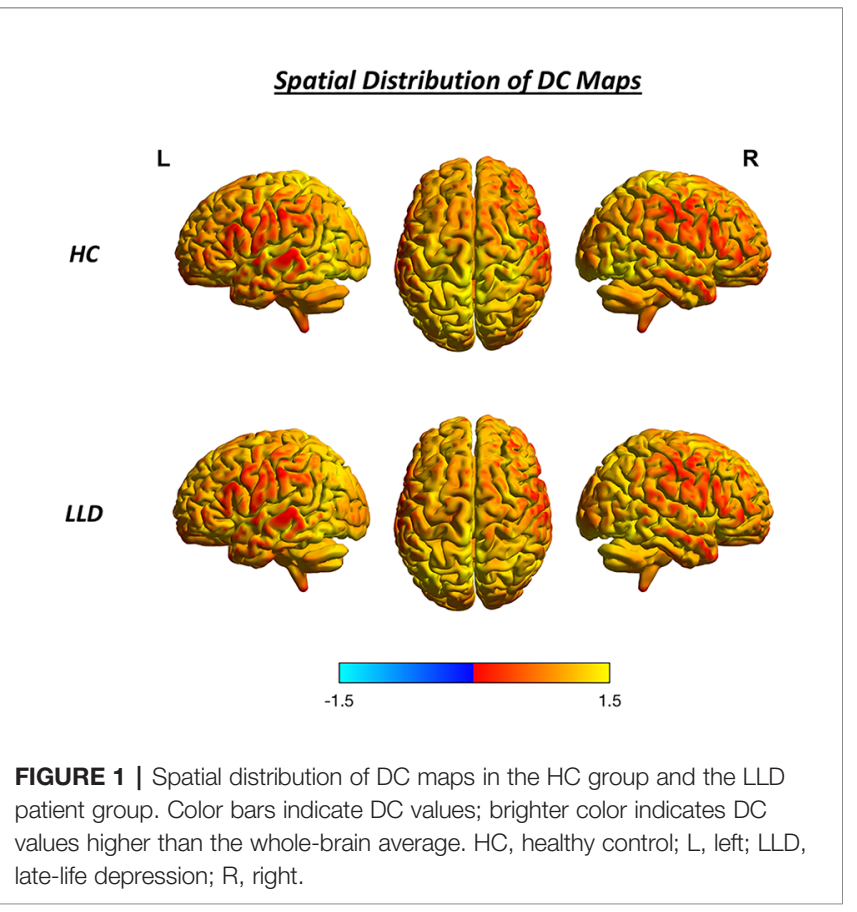

\section{DISCUSSION}

In the present study, the voxel-wise whole-brain functional connectivity in patients with late-life depression was explored using resting-state fMRI techniques. We found a unique pattern of alterations in the brain activity of the patients. Centrality indices,
TABLE 2 | Brain areas showing different DC between LLD patients and HCs $(p<0.05$, corrected).

\begin{tabular}{lccccc} 
Region (AAL name) & \multicolumn{3}{c}{ Peak MNI coordinate } & Voxel size & Peak $\boldsymbol{T}$ value \\
\cline { 2 - 4 } & $\boldsymbol{x}$ & $\boldsymbol{y}$ & $\boldsymbol{z}$ & & \\
\cline { 2 - 4 } & & & & & \\
LLD > HC & 54 & -45 & 54 & 13 & 4.94 \\
Parietal_Inf_R & 15 & -15 & -27 & 29 & 5.21 \\
Parahippocampal Gyrus_R & 3 & -39 & -33 & 19 & 4.42 \\
Brainstem_R/L & -3 & -84 & -27 & 19 & 4.71 \\
Cerebelum_Crus2_L & & & & & \\
LLD < HC & -21 & -42 & 57 & 17 & -4.27 \\
Postcentral_L & -24 & -30 & 54 & 13 & -5.44 \\
Precentral_L & -24 & -69 & -57 & 13 & -4.22 \\
Cerebelum_8_L & & &
\end{tabular}

$A A L$, automated anatomical labeling; $H C$, healthy control; $L$, left; $L L D$, late-life depression; MNI, Montreal Neurological Institute; $R$, right.

measured by voxel-wise degree centrality, were found to be abnormal in late-life depressive patients in the somatosensorymotor areas, inferior parietal lobule, parahippocampal gyrus, cerebellum, and brainstem. Furthermore, differentiated cortical areas with DC values were observed in the LOD patients compared with the EOD patients, in the posterior temporal gyrus and occipital region. No correlation was found between the abnormal centrality indices and the clinical assessment in the patients. The general function of brain areas with an abnormal degree centrality in the LLD patients, as well as the difference between EOD and LOD subgroups, may strengthen the understanding of the intrinsic neural-network profiles of LLD.

The somatosensory cortex receives all sensory inputs from the body, and it is responsible for somatosensory perception (40).

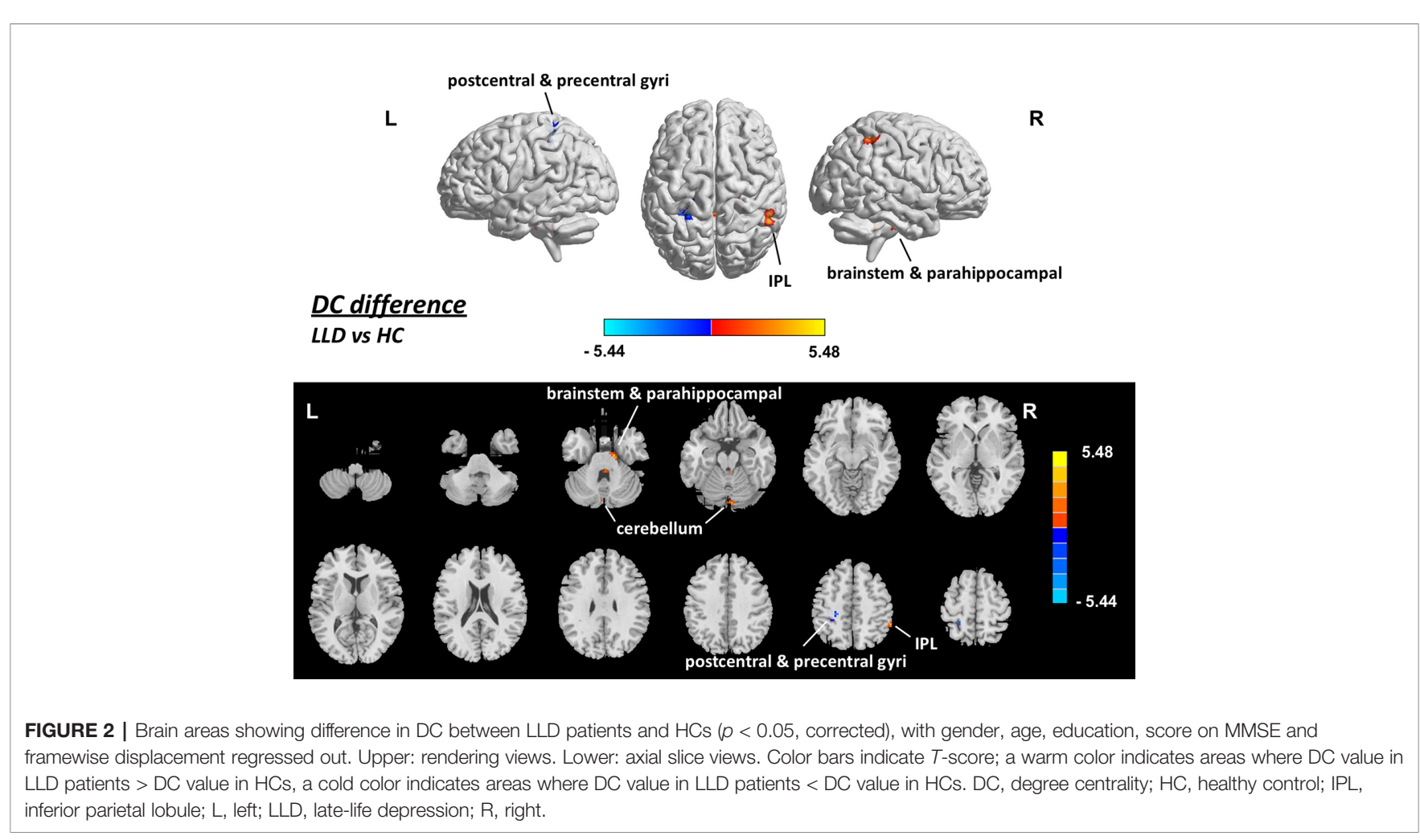




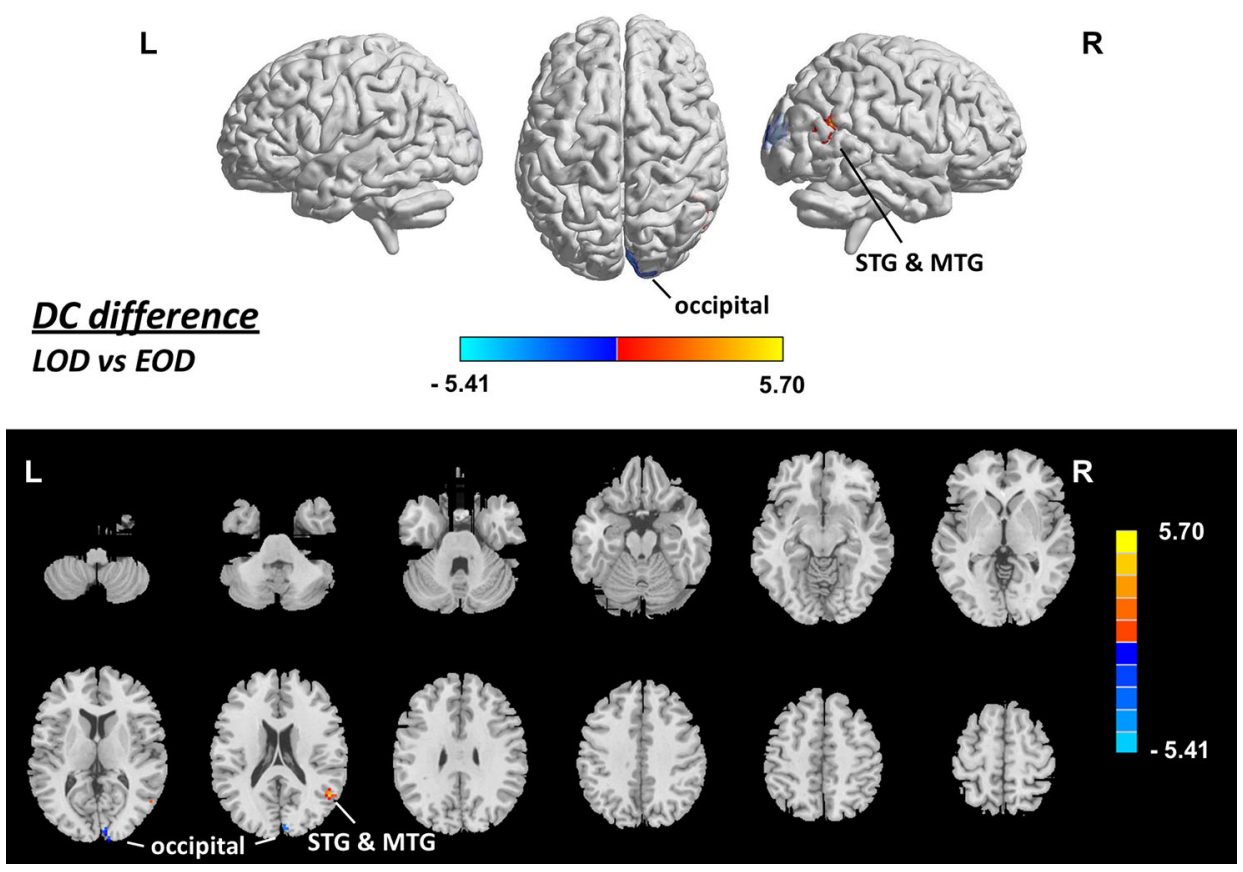

FIGURE 3 | Brain areas showing difference in DC between LOD and EOD patients ( $p<0.05$, corrected), with gender, age, education, score on MMSE and framewise displacement regressed out. Upper: rendering views. Lower: axial slice views. Color bars indicate $T$-score; warm color indicates areas whose DC value in LOD patients > DC value in EOD patients, cold color indicates areas whose DC value in LOD patients < DC value in EOD patients. EOD, early-onset depression; HC, healthy control; L, left; LOD, late-onset depression; MTG, middle temporal gyrus; R, right; STG, superior temporal gyrus.

TABLE 3 | Brain areas showing different DC between LOD and EOD patients $(p<0.05$, corrected).

\begin{tabular}{lccccc}
\hline Region (AAL name) & \multicolumn{2}{l}{ Peak MNI coordinate } & Voxel size & Peak $\boldsymbol{T}$ value \\
\cline { 2 - 3 } & $\boldsymbol{x}$ & $\boldsymbol{y}$ & $\boldsymbol{z}$ & & \\
\hline LOD > EOD & & & & & \\
Temporal_Sup_R & 51 & -57 & 21 & 13 & 5.27 \\
$\begin{array}{l}\text { Temporal_Mid_R } \\
\text { LOD < EOD }\end{array}$ & 54 & -63 & 12 & 13 & 5.70 \\
Cuneus_R & 12 & -93 & 12 & 36 & -5.41 \\
\end{tabular}

$A A L$, automated anatomical labeling; $E O D$, early-onset depression; $L$, left; $L O D$, late-onset depression; MNI, Montreal Neurological Institute; $R$, right.

Abnormal somatic symptoms such as somatization, defined as physical symptoms developed as a result of stress or emotional problems, have often been observed in depressive patients (41, 42). Alteration of activity in the somatosensory cortex has been suggested to be involved in the neural underpinnings of somatic symptom disorder (43). The motor cortex and cerebellum are regarded as important brain regions related to voluntary movement and motor control $(44,45)$. Deficits in motorrelated functions have recently been observed in LLD patients (46). Thus, our observation of decreased DC in somatosensorymotor cortices and altered cerebellar DC in the LLD patients might be related to the somatosensory abnormalities and motor deficits associated with depression.

The inferior parietal lobule, a major network hub of the human brain, is involved in a broad range of behaviors and functions from bottom-up perception to social cognition and plays as important nodes in multiple network, including the frontoparietal control network, default mode network, cingulo-opercular network and ventral attention network (47). A resting-state fMRI study indicated that the connectivity between the dorsomedial prefrontal cortex and the inferior parietal lobule is related with negative self-focused thought associated with depressive symptoms in the patients with major depression (48). Our observation of increased DC at inferior parietal lobule indicates an intrinsic functional alteration in this parietal hub area in the LLD patients.

The parahippocampal gyrus is a limbic structure mainly associated with visuospatial processing and episodic memory (49). Previous studies have revealed that LLD is associated with impaired visuospatial memory and episodic memory $(50,51)$. The white matter integrity of parahippocampal gyrus was found disrupted in the LLD patients (52). An fMRI study indicated abnormal activation of parahippocampal gyrus while performing a memory task in LLD patients (53). Another study including 1,017 participants from the Human Connectome Project revealed that increased functional connectivity of the parahippocampal gyrus is associated with poor sleep quality and depressive problems scores (54). Thus, the increased centrality in parahippocampal gyrus observed in the present study may be related with impaired memory and depressive symptoms and poor sleep quality in the LLD patients.

The brainstem is an important structure that regulates autonomic functions, relays sensory and motor information, and 
modulates cognition, mood, and emotions. The brainstem is particularly critical in the modulation of emotion, as it is the home to a group of modulatory neurotransmitters such as serotonin, dopamine, and norepinephrine (55). Imaging studies have reported structural and functional abnormalities in the brainstem of patients with major depression $(56,57)$. Some studies also indicate that the brainstem aminergic nuclei is closely associated with late-life depressive symptoms $(58,59)$. Our observation of decreased network centrality of brainstem confirms an intrinsic functional abnormality of brainstem nuclei in the late-life depressive individuals.

Besides the brain areas with abnormal DC between LLD patients and HCs, we also observed DC differences between LOD patients and EOD patients, which supports the perspective that the LOD patients and EOD patients have differentiated intrinsic brain networks. The posterior middle temporal gyrus has been suggested to play critical roles in the integration of automatic information retrieval and executively-demanding goal-oriented cognition (60). The occipital cortex is mainly responsible for visual stimulus processing (61). Some studies have revealed that LOD patients have more extensive deficits than EOD patients in some cognitive domains, including the realm of memory and visualspatial processing (27-29). In the present study, brain areas of posterior temporal gyrus and occipital areas were found with different DC values in the LOD patients and the EOD patients. It is tempting to assume that the differentiated levels of DC may be associated with the neural basis for different degrees of impairment in high cognition between LOD and EOD patients.

The study had several limitations. First, the negative result of the relationship of DC with clinical assessment (depression severity, duration of depression, and onset age) indicates that the abnormality in DC might not directly contributed to depressionrelated manifestations, and suggests that the abnormality might be associated with the somatic and emotional burden, and cognitive deficits other than depression itself. However, the resting-state fMRI was performed without tasks measuring emotional and cognitive processing in the patients. In future studies, the relationship of abnormal centrality indices and the emotional processing/cognitive functions needs to be examined by introducing tasks being tested in the patients. Second, although the abnormality of DC in LLD patients has been identified, the sample size of the present study is relatively small. The negative effects of DC difference in the prefrontal regions and the negative result of the relationship between DC and clinical assessment indicates may be attributed to the relatively small sample size. The findings need to be confirmed in future studies using large sample sizes. Third, the antidepressants the patients took may affect the resting-state brain activity. Our findings also did not assess the role of medicine elution which may contribute to the abnormality of the brain-network profile of the patients.

\section{CONCLUSION}

Our findings in the present study indicate that the voxel-wise DC displays abnormality in LLD patients in a wide range of brain areas, which might be associated with the sensorimotor- and emotion-related alterations, and cognitive impairments observed in the patients. Also, there exists a difference in DC patterns between EOD and LOD patients. Our study might help to strengthen the understanding of the pathophysiology of LLD.

\section{DATA AVAILABILITY STATEMENT}

The datasets generated for this study are available on request to the corresponding authors.

\section{ETHICS STATEMENT}

The studies involving human participants were reviewed and approved by Ethics Committee of Shanghai Pudong New Area Mental Health Center. The patients/participants provided their written informed consent to participate in this study.

\section{AUTHOR CONTRIBUTIONS}

HG, YH, YJ, CZ, VV, BS, FY and SZ conceived and designed the study. $\mathrm{HG}, \mathrm{YH}$ and $\mathrm{YJ}$ recruited the participants. QD and $\mathrm{NH}$ collected the data. QD, NH, JL and HX analyzed the data. JL, HG, $\mathrm{HX}, \mathrm{CZ}, \mathrm{VV}, \mathrm{BS}, \mathrm{FY}$ and SZ interpreted the data and wrote the paper.

\section{FUNDING}

This work was supported by the National Natural Science Foundation of China (81771482 to BS); Shanghai Science and Technology Committee (18410710400 to BS); Shanghai Pudong New District Health and Family Planning Commission Key Discipline Construction Fund Project (PWZxk2017-29); Outstanding Clinical Discipline Project of Shanghai Pudong (PWYgy2018-10). Dr. VV is supported by a Medical Research Council Senior Clinical Fellowship (MR/P008747/1). The funding organizations played no further role in study design, data collection, analysis and interpretation and paper writing.

\section{ACKNOWLEDGMENTS}

The authors thank all the individuals who served as the research participants.

\section{SUPPLEMENTARY MATERIAL}

The Supplementary Material for this article can be found online at: https://www.frontiersin.org/articles/10.3389/fpsyt.2019. 01024/full\#supplementary-material 


\section{REFERENCES}

1. BirrerRB, VemuriSP. Depression in later life: a diagnostic and therapeutic challenge. Am Fam Physician (2004) 69(10):2375-82.

2. RiddleM, Potter GG, McQuoid DR, Steffens DC, Beyer JL, Taylor WD. Longitudinal cognitive outcomes of clinical phenotypes of late-life depression. Am J Geriatr Psychiatry (2017) 25(10):1123-34. doi: 10.1016/ j.jagp.2017.03.016

3. Maeshima H, Baba H, Nakano Y, Satomura E, Namekawa Y, Takebayashi N, et al. Time course for memory dysfunction in early-life and late-life major depression: a longitudinal study from the juntendo university mood disorder project. J Affect Disord (2013) 151(1):66-70. doi: 10.1016/j.jad.2013.05.050

4. Liao W, Zhang X, Shu H, Wang Z, Liu D, Zhang Z. The characteristic of cognitive dysfunction in remitted late life depression and amnestic mild cognitive impairment. Psychiatry Res (2017) 251:168-75. doi: 10.1016/ j.psychres.2017.01.024

5. Dybedal GS, Tanum L, Sundet K, Gaarden TL, Bjolseth TM. Neuropsychological functioning in late-life depression. Front Psychol (2013) 4:381. doi: 10.3389/fpsyg.2013.00381

6. Potter GG, Madden DJ, Costello MC, Steffens DC. Reduced comparison speed during visual search in late life depression. J Clin Exp Neuropsychol (2013) 35 (10):1060-70. doi: 10.1080/13803395.2013.856381

7. Rajtar-Zembaty A, Salakowski A, Rajtar-Zembaty J, Starowicz-Filip A. Executive dysfunction in late-life depression. Psychiatr Pol (2017) 51 (4):705-18. doi: 10.12740/PP/OnlineFirst/63765

8. Schweitzer I, Tuckwell V, Ames D, O'Brien J. Structural neuroimaging studies in late-life depression: a review. World J Biol Psychiatry (2001) 2(2):83-8. doi: $10.3109 / 15622970109027497$

9. Du M, Liu J, Chen Z, Huang X, Li J, Kuang W, et al. Brain grey matter volume alterations in late-life depression. J Psychiatry Neurosci (2014) 39(6):397-406. doi: $10.1503 /$ jpn. 130275

10. Aizenstein HJ, Butters MA, Wu M, Mazurkewicz LM, Stenger VA, Gianaros PJ, et al. Altered functioning of the executive control circuit in late-life depression: episodic and persistent phenomena. Am J Geriatr Psychiatry (2009) 17(1):30-42. doi: 10.1097/JGP.0b013e31817b60af

11. Leal SL, Noche JA, Murray EA, Yassa MA. Disruption of amygdalaentorhinal-hippocampal network in late-life depression. Hippocampus (2017) 27(4):464-76. doi: 10.1002/hipo.22705

12. Eyre HA, Yang H, Leaver AM, Van Dyk K, Siddarth P, Cyr NS, et al. Altered resting-state functional connectivity in late-life depression: a cross-sectional study. J Affect Disord (2016) 189:126-33. doi: 10.1016/j.jad.2015.09.011

13. Cieri F, Esposito R, Cera N, Pieramico V, Tartaro A, di Giannantonio M. Latelife depression: modifications of brain resting state activity. J Geriatr Psychiatry Neurol (2017) 30(3):140-50. doi: 10.1177/0891988717700509

14. Liu F, Hu M, Wang S, Guo W, Zhao J, Li J, et al. Abnormal regional spontaneous neural activity in first-episode, treatment-naive patients with late-life depression: a resting-state fMRI study. Prog Neuropsychopharmacol Biol Psychiatry (2012) 39(2):326-31. doi: 10.1016/j.pnpbp.2012.07.004

15. Zuo XN, Ehmke R, Mennes M, Imperati D, Castellanos FX, Sporns O, et al. Network centrality in the human functional connectome. Cereb Cortex (2012) 22(8):1862-75. doi: 10.1093/cercor/bhr269

16. Liu F, Zhu C, Wang Y, Guo W, Li M, Wang W, et al. Disrupted cortical hubs in functional brain networks in social anxiety disorder. Clin Neurophysiol (2015) 126(9):1711-6. doi: 10.1016/j.clinph.2014.11.014

17. Guo Z, Liu X, Hou H, Wei F, Liu J, Chen X. Abnormal degree centrality in Alzheimer's disease patients with depression: A resting-state functional magnetic resonance imaging study. Exp Gerontol (2016) 79:61-6. doi: 10.1016/j.exger.2016.03.017

18. Eijlers AJ, Meijer KA, Wassenaar TM, Steenwijk MD, Uitdehaag BM, Barkhof $\mathrm{F}$, et al. Increased default-mode network centrality in cognitively impaired multiple sclerosis patients. Neurology (2017) 88(10):952-60. doi: 10.1212/ WNL.0000000000003689

19. Zhou Q, Womer FY, Kong L, Wu F, Jiang X, Zhou Y, et al. Trait-related corticalsubcortical dissociation in bipolar disorder: analysis of network degree centrality. J Clin Psychiatry (2017) 78(5):584-91. doi: 10.4088/JCP.15m10091

20. Wang H, Zhang B, Zeng B, Tang Y, Zhang T, Zhao S, et al. Association between catechol-O-methyltransferase genetic variation and functional connectivity in patients with first-episode schizophrenia. Schizophr Res (2018b) 199:214-20. doi: 10.1016/j.schres.2018.04.023

21. Liu F, Tian H, Li J, Li S, Zhuo C. Altered voxel-wise gray matter structural brain networks in schizophrenia: Association with brain genetic expression pattern. Brain Imaging Behav (2019) 13(2):493-502. doi: 10.1007/s11682-0189880-6

22. Zhou M, Yang C, Bu X, Liang Y, Lin H, Hu X, et al. Abnormal functional network centrality in drug-naive boys with attention-deficit/hyperactivity disorder. Eur Child Adolesc Psychiatry (2019) 28(10):1321-8. doi: 10.1007/ s00787-019-01297-6

23. Di Martino A, Zuo XN, Kelly C, Grzadzinski R, Mennes M, Schvarcz A, et al. Shared and distinct intrinsic functional network centrality in autism and attention-deficit/hyperactivity disorder. Biol Psychiatry (2013) 74(8):623-32. doi: 10.1016/j.biopsych.2013.02.011

24. Wang $\mathrm{H}$, Chen $\mathrm{H}$, Wu J, Tao L, Pang Y, Gu M, et al. Altered resting-state voxel-level whole-brain functional connectivity in depressed Parkinson's disease. Parkinsonism Relat Disord (2018a) 50:74-80. doi: 10.1016/ j.parkreldis.2018.02.019

25. Li M, Das T, Deng W, Wang Q, Li Y, Zhao L, et al. Clinical utility of a short resting-state MRI scan in differentiating bipolar from unipolar depression. Acta Psychiatr Scand (2017) 136(3):288-99. doi: 10.1111/acps.12752

26. Grayson L, Thomas A. A systematic review comparing clinical features in early age at onset and late age at onset late-life depression. J Affect Disord (2013) 150(2):161-70. doi: 10.1016/j.jad.2013.03.021

27. Sachs-Ericsson N, Corsentino E, Moxley J, Hames JL, Rushing NC, Sawyer K, et al. A longitudinal study of differences in late- and early-onset geriatric depression: depressive symptoms and psychosocial, cognitive, and neurological functioning. Aging Ment Health (2013) 17(1):1-11. doi: 10.1080/13607863.2012.717253

28. Mackin RS, Nelson JC, Delucchi KL, Raue PJ, Satre DD, Kiosses DN, et al. Association of age at depression onset with cognitive functioning in individuals with late-life depression and executive dysfunction. Am J Geriatr Psychiatry (2014) 22(12):1633-41. doi: 10.1016/j.jagp.2014.02.006

29. Hashem AH, Nasreldin M, Gomaa MA, Khalaf O. O. Late versus early onset depression in elderly patients: vascular risk and cognitive impairment. Curr Aging Sci (2017) 10(3)211-6. doi: 10.2174/1874609810666170404105634

30. Lloyd AJ, Ferrier IN, Barber R, Gholkar A, Young AH, O'Brien JT. Hippocampal volume change in depression: late- and early-onset illness compared. Br J Psychiatry (2004) 184:488-95. doi: 10.1192/bjp.184.6.488

31. Hickie I, Naismith S, Ward PB, Turner K, Scott E, Mitchell P, et al. Reduced hippocampal volumes and memory loss in patients with early- and late-onset depression. Br J Psychiatry (2005) 186:197-202. doi: 10.1192/bjp.186.3.197

32. Janssen J, Hulshoff Pol HE, de Leeuw FE, Schnack HG, Lampe IK, Kok RM, et al. Hippocampal volume and subcortical white matter lesions in late life depression: comparison of early and late onset depression. J Neurol Neurosurg Psychiatry (2007) 78(6):638-40. doi: 10.1136/jnnp.2006.098087

33. Ballmaier M, Narr KL, Toga AW, Elderkin-Thompson V, Thompson PM, Hamilton L, et al. Hippocampal morphology and distinguishing late-onset from early-onset elderly depression. Am J Psychiatry (2008) 165(2):229-37. doi: 10.1176/appi.ajp.2007.07030506

34. Disabato BM, Morris C, Hranilovich J, D'Angelo GM, Zhou G, Wu N, et al. Comparison of brain structural variables, neuropsychological factors, and treatment outcome in early-onset versus late-onset late-life depression. Am J Geriatr Psychiatry (2014) 22(10):1039-46. doi: 10.1016/j.jagp.2013.02.005

35. Lebedeva A, Borza T, Haberg AK, Idland AV, Dalaker TO, Aarsland D, et al. Neuroanatomical correlates of late-life depression and associated cognitive changes. Neurobiol Aging (2015) 36(11):3090-9. doi: 10.1016/ j.neurobiolaging.2015.04.020

36. Chen JD, Liu F, Xun GL, Chen HF, Hu MR, Guo XF, et al. Early and late onset, first-episode, treatment-naive depression: same clinical symptoms, different regional neural activities. J Affect Disord (2012) 143(1-3):56-63. doi: 10.1016/ j.jad.2012.05.025

37. Guo WB, Liu F, Xun GL, Hu MR, Guo XF, Xiao CQ, et al. Reversal alterations of amplitude of low-frequency fluctuations in early and late onset, firstepisode, drug-naive depression. Prog Neuropsychopharmacol Biol Psychiatry (2013) 40:153-9. doi: 10.1016/j.pnpbp.2012.08.014

38. Hamilton M. A rating scale for depression. J Neurol Neurosurg Psychiatry (1960) 23:56-62. doi: 10.1136/jnnp.23.1.56 
39. Folstein MF, Folstein SE, McHugh PR. "Mini-mental state". A practical method for grading the cognitive state of patients for the clinician. J Psychiatr Res (1975) 12(3):189-98. doi: 10.1016/0022-3956(75)90026-6

40. Harding-Forrester S, Feldman DE. Somatosensory maps. Handb Clin Neurol (2018) 151:73-102. doi: 10.1016/B978-0-444-63622-5.00004-8

41. Chakraborty K, Avasthi A, Grover S, Kumar S. Functional somatic complaints in depression: an overview. Asian J Psychiatr (2010) 3(3):99-107. doi: 10.1016/ j.ajp.2010.07.003

42. Bekhuis E, Boschloo L, Rosmalen JG, de Boer MK, Schoevers RA. The impact of somatic symptoms on the course of major depressive disorder. J Affect Disord (2016) 205:112-8. doi: 10.1016/j.jad.2016.06.030

43. Li Q, Xiao Y, Li Y, Li L, Lu N, Xu Z, et al. Altered regional brain function in the treatment-naive patients with somatic symptom disorder: a resting-state fMRI study. Brain Behav (2016) 6(10):e00521. doi: 10.1002/brb3.521

44. Ebbesen CL, Brecht M. Motor cortex - to act or not to act? Nat Rev Neurosci (2017) 18(11):694-705. doi: 10.1038/nrn.2017.119

45. D'Angelo E. Physiology of the cerebellum. Handb Clin Neurol (2018) 154:85108. doi: 10.1016/B978-0-444-63956-1.00006-0

46. O'Brien JT, Gallagher P, Stow D, Hammerla N, Ploetz T, Firbank M, et al. A study of wrist-worn activity measurement as a potential real-world biomarker for late-life depression. Psychol Med (2017) 47(1):93-102. doi: 10.1017/ S0033291716002166

47. Igelstrom KM, Graziano MSA. The inferior parietal lobule and temporoparietal junction: a network perspective. Neuropsychologia (2017) 105:70-83. doi: 10.1016/j.neuropsychologia.2017.01.001

48. Philippi CL, Cornejo MD, Frost CP, Walsh EC, Hoks RM, Birn R, et al. Neural and behavioral correlates of negative self-focused thought associated with depression. Hum Brain Mapp (2018) 39(5):2246-57. doi: 10.1002/hbm.24003

49. Aminoff EM, Kveraga K, Bar M. The role of the parahippocampal cortex in cognition. Trends Cognit Sci (2013) 17(8):379-90. doi: 10.1016/ j.tics.2013.06.009

50. Herrmann LL, Goodwin GM, Ebmeier KP. The cognitive neuropsychology of depression in the elderly. Psychol Med (2007) 37(12):1693-702. doi: 10.1017/ S0033291707001134

51. Klojcnik M, Kavcic V, Bakracevic Vukman K. Relationship of depression with executive functions and visuospatial memory in elderly. Int J Aging Hum Dev (2017) 85(4):490-503. doi: 10.1177/0091415017712186

52. Guo W, Liu F, Xun G, Hu M, Guo X, Xiao C, et al. Disrupted white matter integrity in first-episode, drug-naive, late-onset depression. J Affect Disord (2014) 163:70-5. doi: 10.1016/j.jad.2014.03.044
53. Weisenbach SL, Kassel MT, Rao J, Weldon AL, Avery ET, Briceno EM, et al. Differential prefrontal and subcortical circuitry engagement during encoding of semantically related words in patients with late-life depression. Int J Geriatr Psychiatry (2014) 29(11):1104-15. doi: 10.1002/gps.4165

54. Cheng W, Rolls ET, Ruan H, Feng J. Functional connectivities in the brain that mediate the association between depressive problems and sleep quality. JAMA Psychiatry (2018) 75(10):1052-61. doi: 10.1001/jamapsychiatry.2018.1941

55. Venkatraman A, Edlow BL, Immordino-Yang MH. The brainstem in emotion: a review. Front Neuroanat (2017) 11:15. doi: 10.3389/fnana.2017.00015

56. Supprian T, Reiche W, Schmitz B, Grunwald I, Backens M, Hofmann E, et al. MRI of the brainstem in patients with major depression, bipolar affective disorder and normal controls. Psychiatry Res (2004) 131(3):269-76. doi: 10.1016/j.pscychresns.2004.02.005

57. Song YJ, Korgaonkar MS, Armstrong LV, Eagles S, Williams LM, Grieve SM. Tractography of the brainstem in major depressive disorder using diffusion tensor imaging. PloS One (2014) 9(1):e84825. doi: 10.1371/ journal.pone.0084825

58. Manji HK, Drevets WC, Charney DS. The cellular neurobiology of depression. Nat Med (2001) 7(5):541-7. doi: 10.1038/87865

59. Wilson RS, Nag S, Boyle PA, Hizel LP, Yu L, Buchman AS, et al. Brainstem aminergic nuclei and late-life depressive symptoms. JAMA Psychiatry (2013) 70(12):1320-8. doi: 10.1001/jamapsychiatry.2013.2224

60. Davey J, Thompson HE, Hallam G, Karapanagiotidis T, Murphy C, De Caso I, et al. Exploring the role of the posterior middle temporal gyrus in semantic cognition: integration of anterior temporal lobe with executive processes. Neuroimage (2016) 137:165-77. doi: 10.1016/j.neuroimage.2016.05.051

61. Wandell BA, Dumoulin SO, Brewer AA. Visual field maps in human cortex. Neuron (2007) 56(2):366-83. doi: 10.1016/j.neuron.2007.10.012

Conflict of Interest: The authors declare that the research was conducted in the absence of any commercial or financial relationships that could be construed as a potential conflict of interest.

Copyright (C) 2020 Li, Gong, Xu, Ding, He, Huang, Jin, Zhang, Voon, Sun, Yan and Zhan. This is an open-access article distributed under the terms of the Creative Commons Attribution License (CC BY). The use, distribution or reproduction in other forums is permitted, provided the original author(s) and the copyright owner(s) are credited and that the original publication in this journal is cited, in accordance with accepted academic practice. No use, distribution or reproduction is permitted which does not comply with these terms. 